\title{
Segmentation Study of Aged Gait Based on FFT
}

\author{
Li Zhu, Yuchuan Wu ${ }^{*}$, Shuangbao Ma and Shengfeng Qi \\ School of Mechanical Engineering and Automation, Wuhan Textile University, Wuhan, Hubei, 430073 \\ ${ }^{*}$ Corresponding author
}

\begin{abstract}
To study the rules and characteristics of the daily actions of the aged, a set of multi-sensor wearable device has been developed. As for the segmenting method of action data collected from the aged wearing this device, the human action sequence segmentation method has been proposed based on the motion characteristics in this paper. That is to say, collecting the data for Fourier transform, determining the equilibrium position of the motion, and then returning back to the time domain and determining the segmentation point by judging the changing trend of signal waveform, so as to realize the segmentation of the entire signal. Experiments show that this method can effectively segment the daily actions of the aged, extract valid action sequence, and is particularly effective in the segmentation of regular actions.
\end{abstract}

Keywords-wearable sensor; motion characteristics; motion data segmentation; FFT

\section{INTRODUCTION}

In the studies of the lower limb motion recognition of the aged, the segmentation of the original motion data is an important par $t$ of the action recognition by the model and the segmentation quality of the data directly affects the precision of the action recognition by the model [1]. The purpose of data segmentation is to segment the collected motion data sequence into the motion segments of a single cycle. One segment refers to an action [2].

At present, there are so many studies of the motion analysis of the aged and various motion data capture methods, which mainly include two classes: one is the image motion capture data based on the video and another one is the disperse motion capture data based on the wearable sensor. The motion study based on the video is the mainstream and the relevant technologies are relatively matured, but the amount of data is so large that it is necessary to reduce the dimensions of the large data matrix [3-6]. This paper adopts the wearable sensor to gather the human motion data sequence. This method is featured in low cost and relatively flexible use and is applicable to various occasions. This method can decrease the data amount under the condition of not changing the action information.

In combination with the class of activity and the related information of limbs and trunk of the aged, it is found in this paper through the observation and statistics of massive sample data that the single changes take place in the data of the flexible sensor in the process of walking and sitting of the aged, thus, the changes in the motion segments of the aged can be determined in the original data. So, this paper determines the motion cycle through the fluctuation equilibrium line of the data of the flexible sensor and determines the segmentation point according to the peak and valley value of the data in the cycle.

\section{COLLECTION AND ANALYSIS OF Motion DATA}

The human motion is a regular motion and it can be repeatedly achieved through the deformation of the limbs and trunk. The study of this paper is to segment the data collected by the wearable system of human motion recognition of multiple sensor. The collection system is composed of inertial sensor placed on the waist and two flexible sensors placed on the knee joints, among which the inertial sensor measures 3D acceleration data reflecting the changes in the gravity center and the inertial direction of the trunk of the human while the flexible sensor measures the data reflecting the change angle of the joints. Any motion action of the lower limbs of the human body can be represented with 3D acceleration data and the data of the flexible sensors placed on the right and left knee.

\section{A. Tester and Action}

8 testers from Wuhan Baoxie Health Service Center (2 men, 6 women, aged $60-70$, height: $155 \sim 175 \mathrm{~cm}$, weight: $50 \sim 80 \mathrm{~kg}$ ) were selected for the test of 6 actions including running, sitting, standing, sleeping, rising and walking respectively.

The acceleration sensor in the wearable sensor device [7] is horizontally placed at the waist and two flexible sensors are respectively fixed at both knees of the tester. The received data are stored in format of txt. There were 5 columns of data, the first three columns were the data of the acceleration sensor at the waist and the rest two columns were the data of the flexible sensors on the knees. Through reading the data with matlab, the signal waveform was represented as follows:

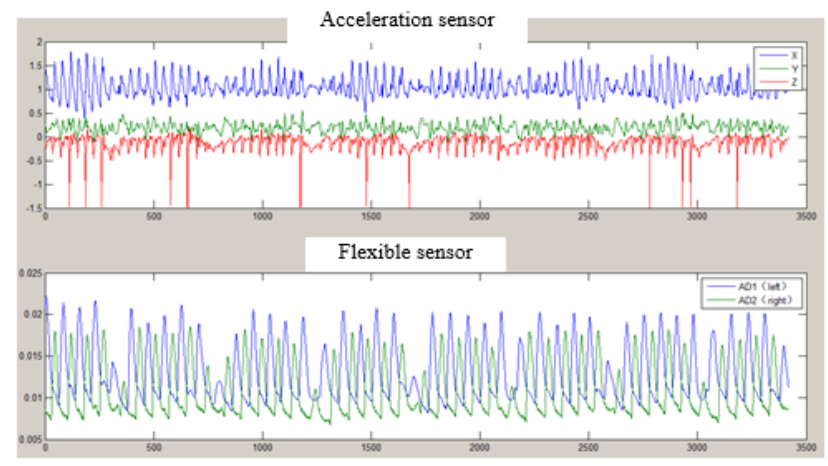

FIGURE I. OSCILLOGRAM OF DATA SIGNAL OF RUNNING 
Where, $\mathrm{x}, \mathrm{y}$ and $\mathrm{z}$ were the acceleration data, $\mathrm{x}$ was the upper and lower inertial direction of the human body, $y$ was the right and left inertial direction of the human body and $\mathrm{Z}$ is the front and back inertial direction of the human body; AD1 and AD2 were the data of the flexible sensors, AD1 was the curvature of the left knee and AD2 was the one of the right knee.

\section{B. Analysis of Human Motion Data}

The motor system, under the domination of the nervous system, with the bone as the lever, with the joint as the pivot and with muscle contraction as the organ power, generates the motion. As to the same action, the change in the gravity center and the one in the motion amplitude of the corresponding lower limb joints are regular and they are interconnected. The roles of different reference data are inconsistent in description of the actions. Therefore, the segmentation point of the whole motion sequence can be determined based on one group of the reference data with the best description performance. The process of the gait was shown in figure II.

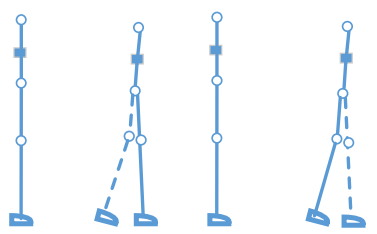

FIGURE II. DECOMPOSITION CHART OF ACTIONS OF THE AGED

In walking or running, with the forward shift of the gravity center, both legs of the human were in the repeated process of being upstanding---bending---upstanding and the most remarkable feature reflected into the data display was that the data of the flexible sensors on both legs were alternately in a cyclic process of the min. value---the max. value---the min. value, as shown in the following figures.

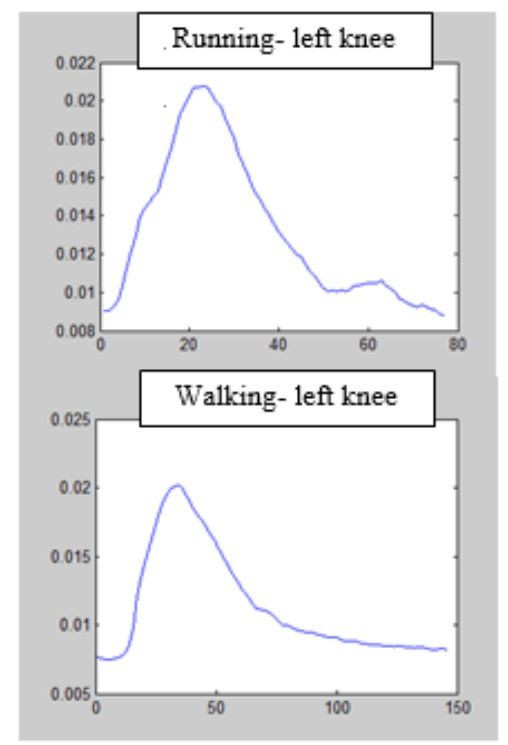

FIGURE III. OSCILLOGRAM OF THE OPTIMAL REFERENCE DATA
As shown in the above figures, as to the actions with a simple cycle, such as walking and sitting, the most remarkable feature was the data of the flexible sensor.

\section{Segmentation of Action SEquence}

The data collected in this paper were collected by the wearable sensor device at the points with the interval of $10 \mathrm{~ms}$, totaling 10-byte data including 2-byte Axis X acceleration, 2byte Axis $\mathrm{Y}$ acceleration, 2-byte Axis $\mathrm{Z}$ acceleration, AD value of 2-byte flexible material 1 and $A D$ value of 2-byte flexible material 2. Every 9 points transmit the data once through wireless after packaging. The data were disperse data, totally 5 columns. Assumed the motion sequence data were $M_{1 j}$, the corresponding time sequence is $T_{j}$, where $M_{1 j}, M_{2 j}$ and $M_{3 y}$ were the motion data of Axis $\mathrm{X}, \mathrm{Y}$ and $\mathrm{Z}$ of $3 \mathrm{D}$ acceleration sensor and $M_{4 j}$ and $M_{B /}$ were the data of AD1 of the left knee and AD2 of the right knee of the flexible sensor.

It could be known through analysis that the optimal data sequence describing both action segments including quick walking and slow walking was from the flexible sensor and presented the signal waveform which was superposed by multiple frequency and similar to sine. As to this class of action, the segmentation point could be determined through directly calculating the peak value and the valley value of the waveform. Assumed the data of the left knee were used as the reference and given $x_{i} \in M_{4 y}, t_{i} \in T_{j}$.

Firstly, Fourier transform was applied to transform the signal waveform from the time domain to the frequency domain. Then:

$$
X_{n}=\sum_{k=0}^{N-1} X_{k} e^{i \frac{2 \pi}{N} \mathrm{kn}} n=0, \ldots ., N-1
$$

Where, $X_{k}$ is the amplitude. In the frequency domain, the position with the frequency of 0 is the equilibrium position of the waveform, denoting the waveform fluctuated up and down around this position.

Secondly, according to the equilibrium position, score one line parallel with Axis $\mathrm{Z}$ in the time domain and it would intersect with the waveform. Apparently, the space between any two continuous points of intersection composed one half of the waveform and the space among three continuous points of intersection composed the whole waveform. Given three points were $x_{i}, x_{j}$ and $x_{k}$, their corresponding time was $t_{i}, t_{j}$ and $t_{k}$. The scope of each waveform could be determined according to the positions of the points of intersection. (The corresponding phase of the position of each point of intersection was the integral multiple of pi).

Calculating the cycle and the amplitude in this scope. Since three continuous points of intersection determined one whole waveform, the cycle and the amplitude could be estimated according to the sample waveform. 
Firstly, the amplitude corresponded to the drop height between the highest point and the lowest point of the waveform.

Therefore, The highest point of waveform:

$$
X_{\mathrm{m}}=\max \left\{x_{i}\right\} \quad i \in[i, k]
$$

The lowest point of waveform:

$$
X_{n}=\min \left\{x_{i}\right\} \quad i \in[i, k]
$$

Amplitude:

$$
A=\frac{\left(X_{m}-X_{\mathrm{n}}\right)}{2}
$$

The cycle of waveform was the time span of a whole waveform, therefore, the cycle was:

$$
T=t_{k}-t_{i}
$$

As to any waveform derived after segmentation, its cycle and amplitude could be calculated. The cycle (amplitude) of a whole waveform could be derived through the mean value of the cycle of each waveform.

\section{EXPERIMENTAL RESULTS AND DISCUSSION}

In order to compare with the accuracy rate of the samples of the automatic segmentation algorithm, the experiment carried out the manual segmentation of the same group of the sample data, recognized both groups of the samples respectively and compared the results.

After isometric processing of the samples after segmentation, this experiment extracted the characteristics of the samples with the wavelet transformation, determined the recognition model with SVM and selected the optimal model with K-fold. Fig. 3 presented the number of the samples involved in the recognition and the selected cross validation.

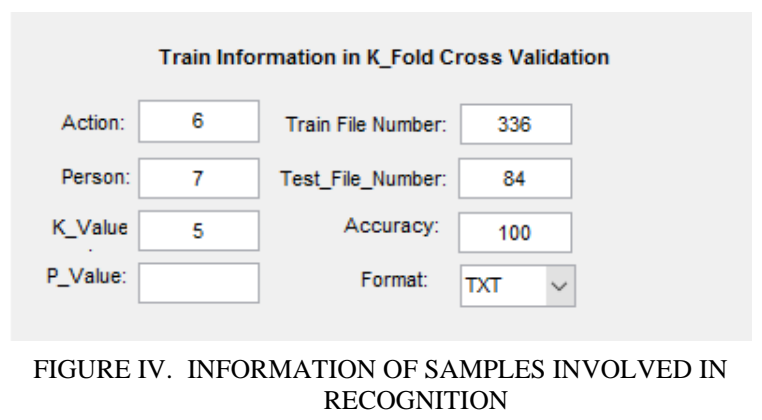

The results of manual segmentation were used as the reference benchmark to compare with the results of the automatic segmentation by means of the motion characteristics. The comparison of both segmentation methods were shown in TABLE I .

TABLE I. RECOGNITION RESULTS OF RECOGNITION DATA

\begin{tabular}{|c|c|c|}
\hline METHODS & $\begin{array}{c}\text { Accuracy rate of } \\
\text { running }\end{array}$ & $\begin{array}{c}\text { Accuracy rate of } \\
\text { walking }\end{array}$ \\
\hline Number of samples & 20 & 20 \\
\hline $\begin{array}{c}\text { manual segmentation } \\
\text { data }\end{array}$ & $50 \%$ & $70 \%$ \\
\hline $\begin{array}{c}\text { automatic } \\
\text { segmentation data }\end{array}$ & $100 \%$ & $75 \%$ \\
\hline
\end{tabular}

It could be seen from the results comparison that the algorithm adopted in this paper could obtain the recognition results of the manual segmentation and was superior over the results of the manual segmentation in the recognition of slow walking and quick walking.

In addition, in the process of experiment, the spurious signal and the interference noise were also found due to the aging characteristics of the testers and the effects of the external environmental factors.

It was found from the experimental results that the method could ignore the effects of the unavoidable noise on the segmentation of the action segments to a relatively great extent.

\section{CONCLUSIONS}

In order to achieve the action recognition, extract the motion parameters such as step size and frequency and establish the motion database, it is necessary to segment the collected motion data as per the motion segment. Based on the characteristics of the flexible sensor in the multiple sensor data collection device, this paper proposed to select one group of parameters reflecting the motion trend to an utmost extent to determine the segmentation point and segment the whole data according to the correlativity of the motion characteristics and the reference data of the actions.

Viewed from the experimental results, the automatic segmentation algorithm was equivalent to even superior over the manual segmentation method. The reason the results appeared was that the methods of manual segmentation and automatic segmentation theoretical selecting the segmentation point were identical with each other, however, since the manual segmentation was firstly affected by the external factors, the actually selected segmentation points might meet with a shift with a certain angle; secondly, the manual segmentation could select the relatively good waveform for segmentation through visual examination while the automatic segmentation was automatically controlled by the program, thus the selection of the segmentation point was more accurate; in addition, the automatic algorithm could segment all of the captured data and remove all of the defective segments according to the motion characteristics, as a result, the motion segments obtained were more accurate in the action recognition effects. 
Also, it could be found in the process of the experiment that the noise in the data signal had a relatively great effect on the automatic segmentation algorithm and it was necessary to find out one more superior method in the late study to remove the effects of the noise to make the segmentation result more accurate and effective.

\section{ACKNOWLEDGMENT}

Funded by the Natural Science Foundation of China No.61271008.

\section{REFERENCES}

[1] Banos, O., et al., Multiwindow Fusion for Wearable Activity Recognition, in Advances In Computational Intelligence, Pt Ii, I. Rojas, G. Joya, and A. Catala, Editors. 2015. p. 290-297.

[2] Liu Xiaoping, Lu Jingting and Xia Xinyu, Motion Segmentation Based on PCA and Mahalanobis Distance, Journal of Hefei University of Technology (Natural Science), 2014. 37: p. 563-566.

[3] Yang Yuedong et al., Segmentation Method of Human Motion Capture Data Based on Geometrical Characteristics, Journal of System Simulation, 2007. 19: p. 2229-2234.

[4] Zhu Dengming and Wang Zhaoqi, Extraction of Keyframe from Motion Capture Data Based on Motion Sequence Segmentation, Journal of Computer-aided Design and Computer Graphics, 2008. 20: p. 787-792.

[5] Shi Xiangbin, et al., Segmentation Method of Motion Capture Data Based on Joint Linkage Characteristics, Journal of System Simulation, 2014. 26(11): p. 2636-2641.

[6] Banos, O., et al., Evaluating the effects of signal segmentation on activity recognition. 2nd International Work-Conference on Bioinformatics and Biomedical Engineering, 2014: p. 759-765.

[7] Wu, Y.-C., et al., Kinematics and dynamics of aged gait wearable sensors. 2nd International Conference for Mechanics and Mechanical Engineering, 2015: p. 150 155. 